\title{
Sensitivity to site and the nature of materials - Southern African architectural design
}

\author{
B. P. Jekot \\ Department of Architecture, University of Pretoria, South Africa
}

\begin{abstract}
This article reports the results of an exercise to review architectural design as the expression of different cultures through materials, where reliance could evolve, pooling different skills and knowledge. This study has been inspired by stunning examples of the so-called 'third' and the 'first' world in Southern African architecture where the inclusion of the 'underdeveloped' in 'developed' technologies can often be seen. Specific environmentally friendly interventions in various socio-cultural and cross-cultural backgrounds are included. The idea behind these designs is to respect the unspoiled natural environment, and to use, where possible, recyclable and natural materials.
\end{abstract}

Keywords: nature of materials, recycling and reuse, technology, identity.

\section{Environmental concerns, climate and design}

Being in the southern hemisphere, Southern African seasons stand in opposition to those of Europe and North America. Famous for its sunshine, Southern Africa is mostly a dry region, receiving only around half of a world's average rainfall, with this rainfall coming predominantly in the summer months. Temperatures in Southern Africa tend to be lower than in other countries at similar latitudes (such as Australia) due mainly to greater elevation above sea level. In winter, for the same reason, nighttime temperatures can drop to below freezing point. The main environmental constraint upon architectural design is the extreme radiation, heat and glare as is common in subtropical climates. The design, therefore, has to respond to this extreme climate. Continuous and efficient ventilation is the primary comfort requirement. Protection from the sun, as well as prevention of internal temperature elevation during the day, should affect all aspects of building design. The layout, planning, as well as the size and location of 
openings/windows are crucial. Open-planning and shared free spaces between buildings help to achieve good ventilation. Openings should be positioned on both windward and leeward sides of buildings to facilitate cross ventilation. Large glazed openings should be protected from sun penetration. In oblique light, screens, shutters and blinds cast thin staggered shadows. Large overhangs work very well in this climate.

Heat and cold weather can be moderated. Thick rock walls can store the coolness from the night air. While during daytime, the rocks absorb and retain the hot air that is then absorbed into the building. Well thought buildings could provide a sustained level of cooling and warming. One can expect six to seven degrees variation from the extremes of outdoor and indoor temperatures. The correct architectural response to this specific climate shapes the identity of the build environment.

\section{The coexistence of the so-called 'third' and the 'first' worlds in Southern African architecture}

The inclusion of the 'underdeveloped' in 'developed' technologies can often be seen in this region. The architecture of the so-called 'third' and 'first' world countries reflects their different characteristics and dynamics: their economical, political, social and cultural dispensations as well as their patterns of collection, production, and consumption. Yet, do 'developed' and 'underdeveloped' technologies have to conflict when it comes to nature and design? Where the nature and resources have been taken into account, the resulting architecture is often both rooted in its environment as well as being functionally relevant and enhances the identity. The power of this combination could be promoted to enrich the way in which we view global coexistence and cooperation as being between partners, rather than between winners and losers. Design should be reviewed as the expression of different cultures where reliance could evolve, pooling different skills and knowledge rooted in nature as bases. The input of information and knowledge should not be imposed but shared.

\section{Tradition, progress and design standards}

It is important to understand the natural environment and its materials in order to create architecture that has roots in its natural environment, functions well and is long-lasting. When nature is used as inspiration, one embraces the values, significance and understanding of varied resources [3]. Bridging nature and design allows for valuable solutions and accomplishments. Such architecture is a statement not just of the patterns of privilege and power, but of established relationships between humans and nature (flora, fauna, materials and other resources). Location has thus defined the nature of architecture. The environment provides a means of expression to the people who call it home and these people have, over time, responded to what was given to them in different ways. Arguments are raised about the appropriateness of exchanging information, technologies and design standards, about how architecture should be designed 
and built or how settlements laid out. When developing the arguments of understanding and responding to nature in order to protect regional identities and culture, we are faced with the issue of applying information and technology transferred from one region to another. These questions are especially pertinent when the move is from a more 'developed' country to a less 'developed' one.

\section{Nature and architectural design - selected samples from Southern Africa}

Southern African contemporary architecture, as illustrated by its variety and character, seems to have benefited greatly from its socio-cultural and crosscultural diversity and the complexity of its context. Contextual issues define bases of architectural design integrated with nature and available resources. Natural materials such as stone, wood, reeds, clay, and recyclables such as glass, metal and tyres are abundant. It is desirable to remove waste from nature and converting this waste into building materials is an ideal situation.

The way in which materials are applied should be fully integrated into the functioning existing order and the values associated with it. If the impetus is to turn what is there into something else and transform it into a 'nobler' version, this has to be done without alienating the local communities, remaining completely connected to nature, while also becoming a distinguishing part of it. The 'underdeveloped' and 'developed' can cooperate creatively responding to the nature and to the nature of materials. Selected samples and applications are going to be illustrated.

\subsection{Nature and indigenous plants}

It is important to understand nature and the regional culture in order to create architecture that has roots in the natural environment. Environmental architecture and indigenous plants can reinforce nature. Location has thus defined the nature of architecture and architectural landscape. Properly selected plants form the identity of contemporary architecture (different types of African grasses) and extend the traditional design (indigenous ivies in Cape Town, well rooted into climate control issues, fig. 1.
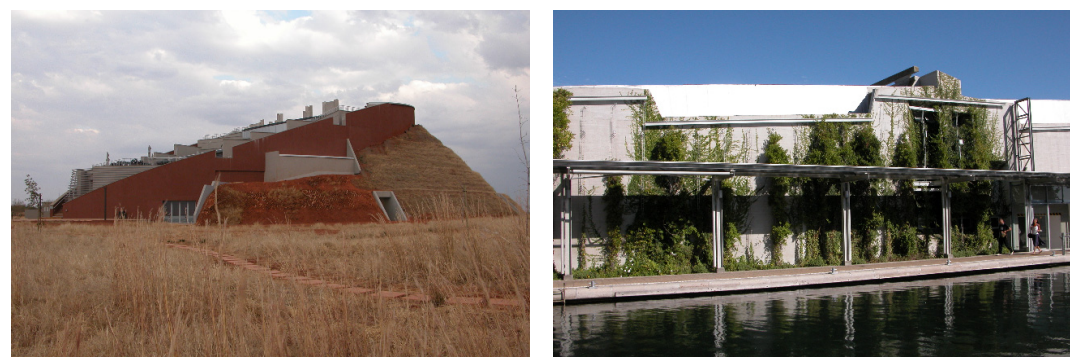

Figure 1: $\quad$ Indigenous plants reinforcing nature, CT and Maropeng, RSA. 


\subsection{Sensitivity to site and reversibility of site}

A strong environmentally sustainable approach was taken to communicate the sensitivity of the site of the Visitors' Centre at a historical rock art location at Twyfelfontein in Namibia [2]. It was important to retain the cultural significance of the area, while respecting the natural landscape. It was also important for any new work to be easily identifiable as such, as well as being removable without damaging the site. Wire caged pebble paths and steps contain no cement were used which allowed for future demolition without waste. Stone gabions and wire cages can be returned to the environment or be recycled in new structures elsewhere. Wire cages and gabions represent the developed technology and hand-crafted steps give the feeling of the natural approach and 'underdeveloped' touch, fig. 2 .
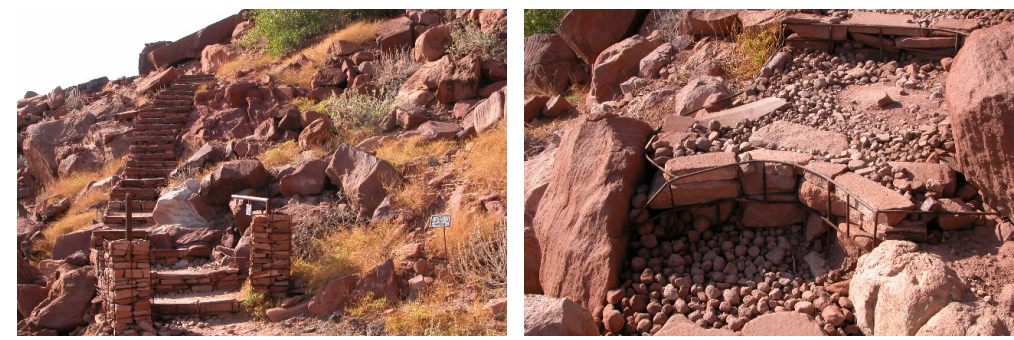

Figure 2: $\quad$ Stone gabions and wire cages, Twyfelfontein, Namibia.

Gabions (wire cages made from standard galvanised diamond mesh) allow one to build solid masonry dry walls. These can be filled with local loose stones gathered from areas in the vicinity, as well as with recycled rubble from old constructions and collected waste. This system, often used in civil engineering projects, is relatively new and can be applied utilising unskilled labour taught on site or professional more sophisticated thin metal structures incorporating different types of meshes, cages and grids plus different types of stone, rocks and fig. 3.
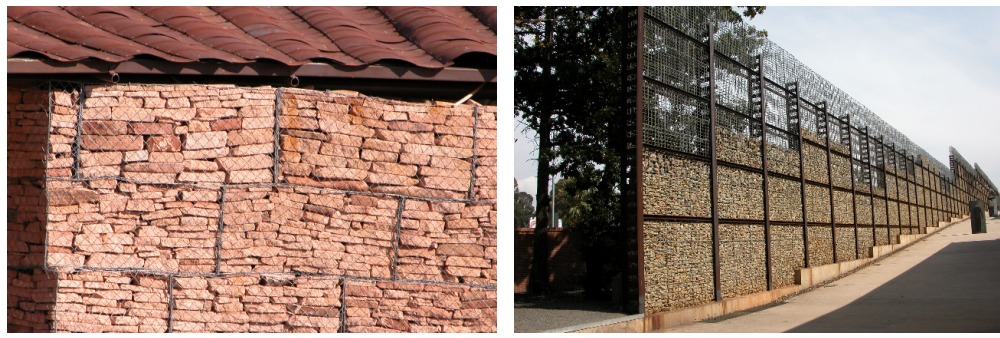

Figure 3: Gabions filled with loose stones and rubble, Namibia and RSA.

The reuse and recycling of materials also leaves room for inventive applications such as a foundation of light structures made from tyres filled with concrete that can be removed as well as light structures, fig. 4 . 

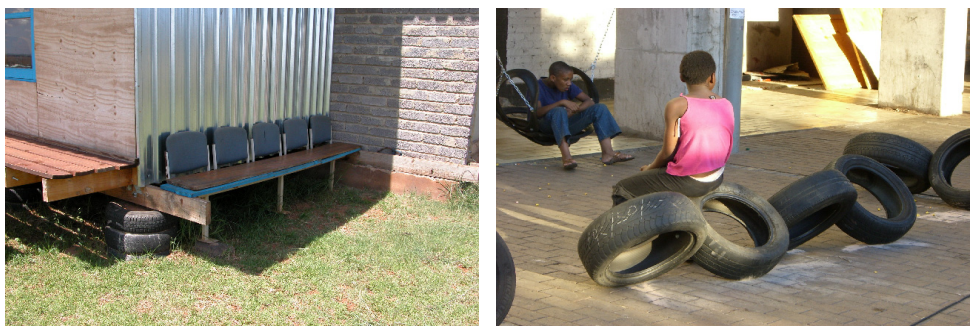

Figure 4: Different applications of tyres, Gauteng, South Africa.

\subsection{Forms and structures}

Many buildings and public places function not only as symbolic landmarks, but in many cases as meeting places for particular users and as bases for community organization. They appear to reflect the legacy of different lifestyles, cultures and skills. Regional communities can adapt 'developed' technologies in their natural settings and create distinctive architecture. Local materials and skills govern the 'developed' technology in Kwa-Zulu Natal and Mapungabwe, South Africa where concrete blocks, representing 'developed' technology, have been used to build the natural oval forms. The gum poles coming from nearby 'undeveloped' neighbourhoods have been applied to columns, the tower, signage and information. This community-based project is a powerful demonstration of cultural identity. It seems the 'undeveloped' can support and inspire 'developed' technologies, fig. 5.
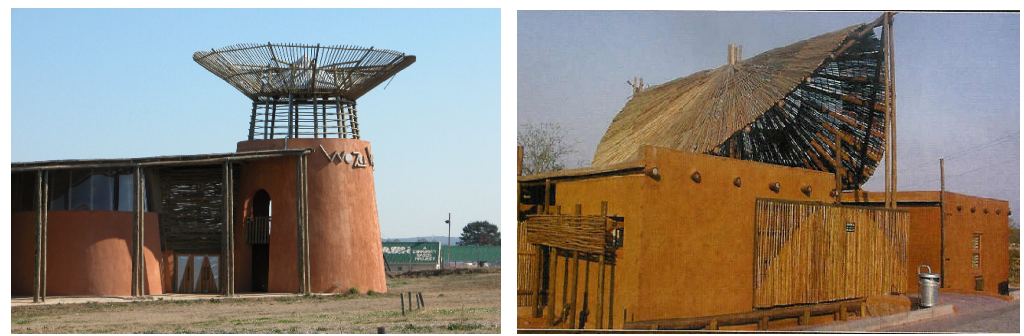

Figure 5: Natural forms, local materials and skills dominate the 'developed'

\subsection{Roofs and shelters}

The beauty of traditional thatched roofing has a very strong feeling of integration with nature. Recently, industrial tiles and sheets have dominated the built environment so strongly that we can hardly imagine something new and innovative. What is new and innovative, however, is the roof cladding consisting of 'tiles' made by quartering recycled oil drums, that exemplifies the spirit of a truly unique place in Twyfelfontein, Namibia where 'underdeveloped' technologies have been flourishing. Cutting the oil drums and reshaping them into large-scale male and female roof tiles has allowed for the creation of sophisticated roof forms that introduce a powerful regional identity, fig. 6 . 

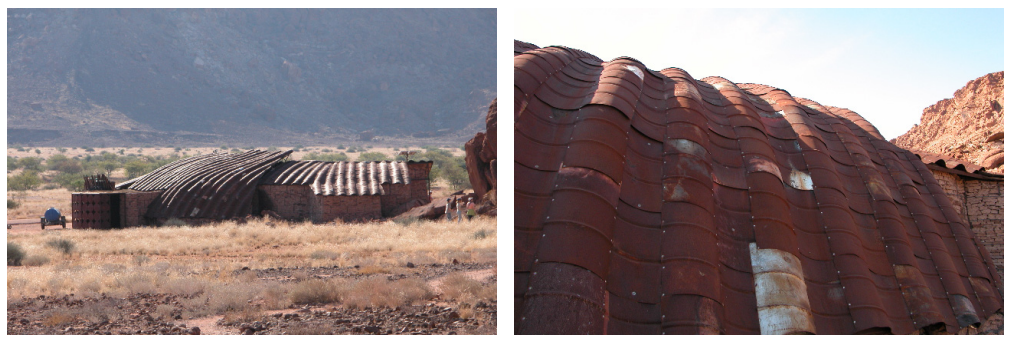

Figure 6: Oil drums converted into huge male/female tiles, Namibia.

The metal was sandblasted before installation to remove old paint and the rusting process has helped blend the building with the red oxides of the rocks all over the site. This natural approach and application of local skills has brought a new perception of how one can utilise ordinary materials, fig. 7.
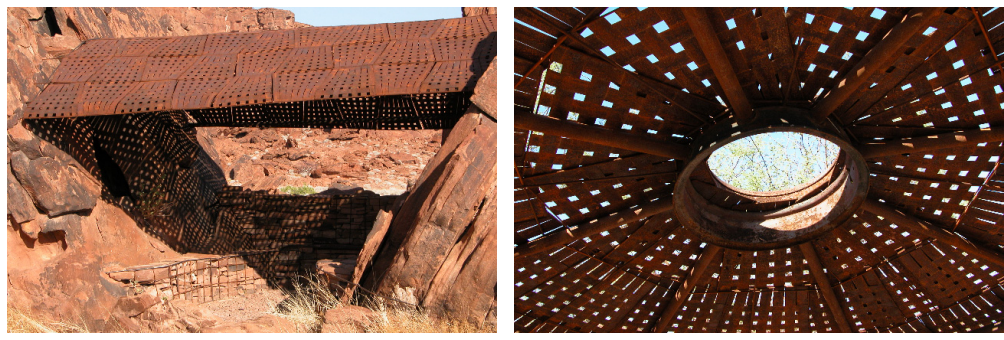

Figure 7: $\quad$ Shelters made of oil drums, Twyfelfontein, Namibia.

\subsection{Light walls, screens, blinds, shutters and fences}

The understanding of the specific context and function of a project can stimulate our innovation. The cheap, light screen walls used in public toilets, for example, show how a usually enduring task can be made less cumbersome by using the available natural materials. Safety and ventilation issues add to the design concept of this architectural example, fig. 8.

If the traditional, completely integrated system is more powerful than the imported corporate one, which has little chance of success in such a place, we have to incorporate what is already there. The design should not seek to impose a foreign and pre-established system - whether it is an architectural style or an architectural language [1]. The details of screens, fences, the decorative ceiling and lighting design of typical industrial parts and ordinary local materials converted into special items, fig. 9. The 'developed' technologies are a canvas for the use of powerful 'underdeveloped' technology, which has been applied thoughtfully and handled vigorously and creatively in many places in Southern Africa's natural environment.

Another example of shaping and forming light walls of materials such as oil drums, tyres and glass bottles is the application of a variety of cans (Coca Cola, Pepsi, Red Bull, beer, et.) into the building process. Walls built using these waste 

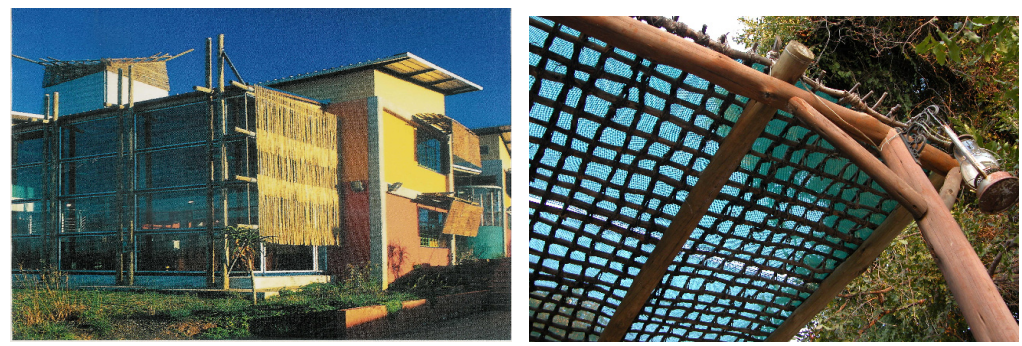

Figure 8: $\quad$ Light walls and screens, Namibia, South Africa.
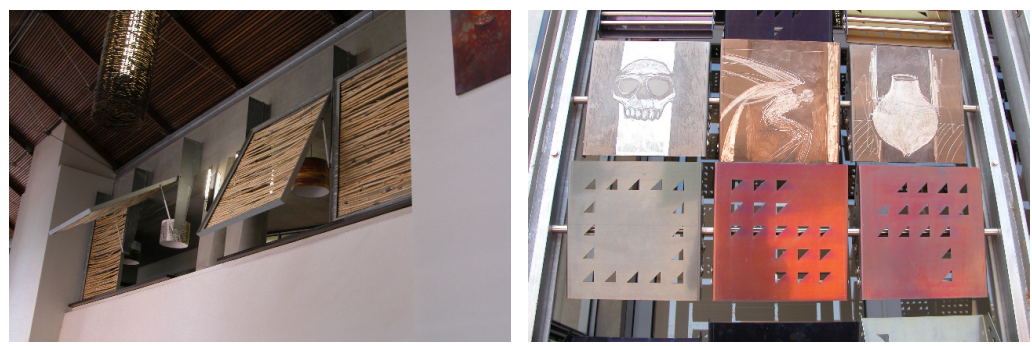

Figure 9: Different types of hand-made screens and blinds, RSA.

materials are light and provide very good insulation. In addition to this, their construction aids in the cleaning of our natural environment from waste products, fig. 10. Use of the branches with thorns from indigenous bushes in the forming of fences and creating boundaries can replace industrial barbed wires.
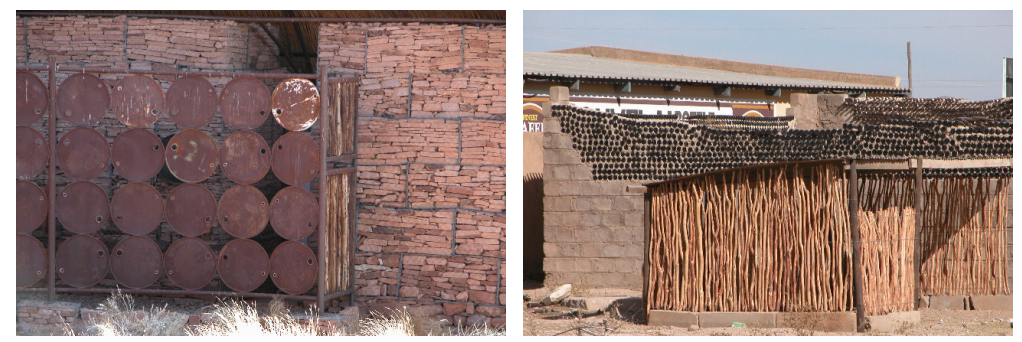

Figure 10: Walls made of glass bottles and tyres, Namibia.

\subsection{Public space and public art inspired by nature}

The colourful South African diversity and the vibrancy of its architecture illustrate its socio-cultural and cross-cultural forces. Public spaces are designed to create different flows to 'market' themselves. Architectural space is unique when it has its own energy because of its conception, its development and its looks, fig. 11. Selected images illustrate the marrying of the functional with public art inspired by nature and the artistic reinterpretation of materials from 
their original functions. Their details show the innovation and creativity expressed, the regional vibe, as well as fun and humour, fig. 12. Their character has usually expanded over a specific time span and from the contributions of various individuals and society. Spaces and buildings document the cultural value composed by different elements from changing flavours. Layer upon layer and experience upon experience have been accumulated, and they in turn influence our well being and future.
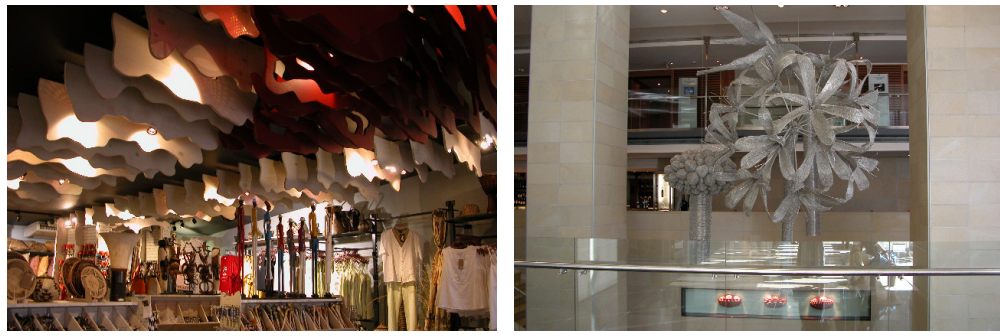

Figure 11: Uniqueness of public space and art applications, RSA.
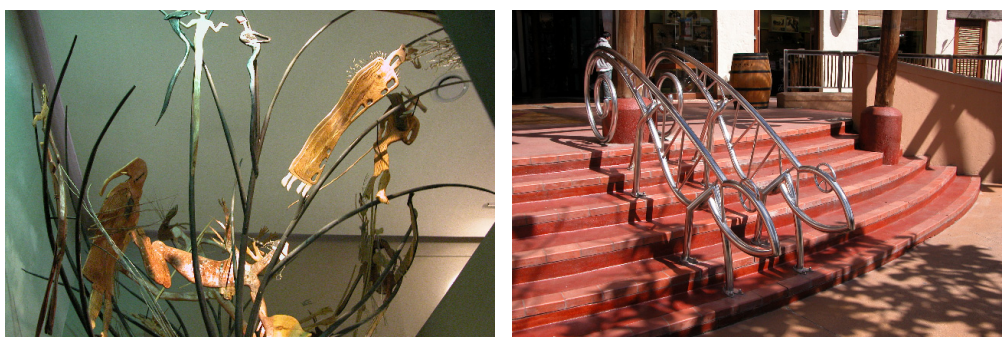

Figure 12: Innovation, creativity, humour and fun, RSA

\section{Redefining the role of architects}

There may be a conflict between natural environment and an increasingly global technological and economic culture. In order to improve the environmental performance of architecture, it is necessary to create a greater awareness of nature and integrate fragmented, contradictory and competing interests and values. There is an increasing demand for higher performance standards in the built environment and for the willingness to deliver such architecture, which in turn requires new, innovative environmental design. Within this context, the extent to which clients, users and designers understand the need, and want to implement policies enforcing sustainable design futures, is extremely important. Architects and designers, through the power of their creativity, help communities around the world embrace change. Today more and more Southern Africans live in cities but the environment they inhabit is very different from what we think of as a traditional European townscape. It is worth exploring the role of nature, identity and expression in African architecture, by looking at the complex 
interplay between climate, context and technology as well as social and environmental processes.

The fabric of architecture is not an art of imposing, but of discerning potentials and bringing them into play. Rather than being constrained, it involves expanded creativity, helping the new to be born and healing what does not work. Many people acknowledge the limits of the human intellect and stress the importance of instinct and intuition. Guided by instinct and intuition rather than by intellect alone, architectural design requires a harmony between head and hand, experience and memory. Instead of the unthinking application of global tendencies, the individual application should grow naturally from the design task, well rooted in the region and its resources.

Southern African spaces and places remind and teach us about the sensitive nature of materials and technologies representing the 'developed' and 'underdeveloped'. The most profoundly rich and dense synthesis of nature and ideas form what is timeless in architecture. This is the mix of simplicity and sophistication of expression, as may be found in the convergence of the so-called 'first' and the 'third' world.

\section{Recapitulation}

Nothing imitative is equal to that which is imitated. Instead of imitating, we should search for the principles that made the imitative original. There is a need and demand for architects who tackle today's requirements and problems with today's solutions and an understanding of nature and its resources. Wellexecuted architecture inspired by nature and integrated with natural resources can make a tremendous contribution to global development and the upliftment of communities.

The generic imposition of an impersonal global design should be replaced by a considered respect for revealed identity. In order to succeed, designers have to reinforce the need for culturally informed architecture. This requires avoiding mere imitation of the vernacular and historical pastiche, and creatively engaging with the living culture of a region and of the world. The contemporary lifestyle and expectations in most of the developed world are unsustainable and unrealistic. Expectations have been raised to inappropriate levels and are giving rise to questions about the distinction between deep moral values and impermanent lifestyles. The need for positive intervention and change at a social and cultural level in many regions is clearly a critical concern in the global movement.

Sharply varying interpretations of sites and exchanging nature of materials and technologies may lead to the designing of environmentally-'progressive' architecture between cultures and societies. The 'developed' and 'undeveloped' can cooperate, creatively finding common sense in nature and appropriate use of available resources. Socio-cultural and cross-cultural transfer is possible, but can be problematic when we do not know why and how to tackle it. The selected sample of architectural interventions illustrate, however, that such transfer is worth trying. It can help us to integrate better and happier humans and enrich 
societies. Architecture that is based on international design standards while sensitively using natural resources is present in Southern Africa.

Contemporary design has had some important positive consequences with respect to regeneration of natural resources. The decentralisation of egalitarian power and sensitive use of our materials, technologies and ideas is needed. Increased environmental degradation and various examples of cultural violence and, of course poverty, has worsened working conditions and inequalities. The question is how we individually address our concerns in many different regions so as to make us stronger and allow us to integrate with nature and create an enhanced identity, well rooted in its natural environment.

\section{References}

[1] Makin, A., In the heart of the cyclone. Light on the Hill: Building the Constitutional Court of RSA, David Krut Publishing, pp 12-31, 2007.

[2] Maritz, N., Visitors' interpretative centre for a prehistoric rock art site at Twyfelfontein, Namibia. Proc. of the annual conference series on Sustainable Built Environment, ed. SBE Archive, pp. 141-150, 2005.

[3] Reader, J., Africa: A Biography of the Continent, Penguin Books, 2003. 